\title{
Preventing postpartum weight retention: Lifestyle interventions in pregnancy, postpartum or both. A systematic review of the literature
}

\author{
K.V. Dalrymple ${ }^{1}$, A.C. Flynn ${ }^{1}$, L. Poston ${ }^{1}$ and M. O’Keeffe ${ }^{2}$ \\ ${ }^{1}$ Department of Women and Children's Health, King's College London, St Thomas' Hospital, London, UK. and \\ ${ }^{2}$ Department of Nutritional Sciences, King's College London, London, UK.
}

This abstract was awarded the student prize for best poster original communication

Obesity is now a global epidemic, with nearly $40 \%$ of the adult population categorised as overweight $(\mathrm{OW})$ or obese $(\mathrm{OB})^{(1)}$. The development of obesity is multifactorial, with increased energy intake and decreased physical activity suggested to be two of the prominent factors ${ }^{(2)}$. Pregnancy may also play a role, as excessive gestational weight gain (GWG) and postpartum weight retention (PPWR) may trigger the development of obesity ${ }^{(3,4)}$. Observational data suggests that women who enter pregnancy overweight or obese are at greater risk of excessive GWG and are less likely to return to their pre-pregnancy weight when compared to women of normal weight ${ }^{(5)}$. The antenatal and postnatal periods are a window of opportunity to prevent obesity in women due to increased contact with healthcare professionals. At present, there is inadequate data to support the implementation of a specific approach to prevent excessive GWG and to reduce PPWR. Therefore, an evidence base for interventions which focus on pregnancy-associated obesity is needed.

The aim of this research was to evaluate the effectiveness of lifestyle interventions in OW/OB pregnant and/or postpartum women at managing PPWR up to 2 years after birth.

A systematic review of the literature was completed from $1^{\text {st }}$ January 2000-23 $3^{\text {rd }}$ January 2018 in MEDLINE, Embase, and CENTRAL for randomised controlled trials within the antenatal and/or postnatal period which reported the effect of diet or diet and physical activity on PPWR up to 2 years after delivery. A hand search of reference lists and cited articles of relevant reports and review articles was also completed.

Electronic searches identified 2753 titles; 35 abstracts were eligible for full-text review. Articles were independently screened by two researchers for relevance, data was then extracted using a structured form and assessed for risk of bias using the Cochrane tool. Fourteen studies $(n=2252)$ were included and divided into three categories, pregnancy only $(n=2)$, postpartum only $(n=9)$, and pregnancy and postpartum $(n=3)$. Recruitment ranged from the first trimester of pregnancy to 18 months postpartum. Intervention duration varied from 10 weeks to 12 months and included either a diet only approach $(\mathrm{n}=4)$ or diet and physical activity $(n=10)$. Type of contact during the intervention also varied from face-to-face $(n=7)$, technology-based $(n=4)$ or both $(n=3)$. Reported weight outcomes ranged from 14 weeks postpartum up to 2 years and included percentage of women below pre-pregnancy weight, change in weight from baseline and PPWR. Of the 14 trials, six from the postpartum period $(\mathrm{n}=363)$ based on short, intensive intervention designs of 10-12 weeks reported significant improvements in PPWR at the end of the interventions and any subsequent follow-up (4-24 months postpartum). The remaining eight trials did not show an effect on long term changes in PPWR. However, four of these trials (pregnancy only $n=2$; pregnancy and postpartum $n=2$ ) reported significant associations between appropriate GWG and reduced PPWR.

Evidence from this review suggests that to reduce PPWR intensive interventions including diet or diet and physical activity, commencing in the postpartum period, are effective. However, due to the heterogeneity of study design, recruitment strategies and small sample sizes, larger trials with similar methodology are required to provide a clear conclusion informing the development of targeted strategies to prevent PPWR.

1. WHO (2014) Fact Sheet $\mathrm{N}^{\circ} 311$.

2. Church T \& Martin CK. (2018) Obesity 26, 14-16.

3. Gaillard R, Durmuş B \& Hofman AI (2013) Obesity 21, 1046-55.

4. Viswanathan M, Siega-Riz AM, Moos MK et al. (2008) Evid Rep Technol Assess 168, 223.

5. Deputy NP, Sharma AJ, Kim SY et al. (2015) Obstet Gynecol. 125, 773. 